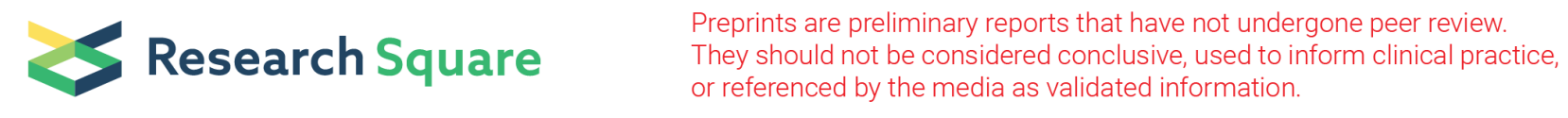

\title{
Meta-analysis of the endothelial protein C receptor rs867186 genotype in malaria
}

\author{
Lin Yang \\ Chongqing Medical and Pharmaceutical University \\ Ruilian Xin \\ Weifang Nursing Vocational College \\ Shanchun Guo \\ Xavier University \\ Mingli Liu ( $\square$ mliu@msm.edu ) \\ Morehouse School of Medicine
}

Research article

Keywords: Severe malaria, cerebral malaria, EPCR, Meta-analysis

Posted Date: June 2nd, 2019

DOI: https://doi.org/10.21203/rs.2.9986/v1

License: (c) (i) This work is licensed under a Creative Commons Attribution 4.0 International License. Read Full License 


\section{Abstract}

The interaction between the P. falciparum erythrocyte membrane protein 1 (PfEMP1) on the surface of parasitized red blood cells (pRBC) and the endothelial cells $(E C)$ receptors during P. falciparum infection results in the sequestration of pRBC from blood circulation. The amount of sequestration is determined by specific interactions among PfEMP1 and several host adhesion receptors, including intercellular adhesion molecule 1 (ICAM-1), CD36, and endothelial protein C receptor (EPCR). PfEMP1 is composed of multiple domains such as the cysteine-rich inter domain region (CIDR) and Duffy binding -like (DBL) domains. CIDRa1 competitively binds to EPCR with activated protein C (APC) and impair cytoprotective and anticoagulant effects by APC, which plays an important role in severe malaria (SM) pathogenesis such as cerebral malaria (CM) and severe malaria anemia (SMA). The strategy to inhibit EPCR binding to pRBC while to concomitantly strengthen its binding to APC may be crucial in restoring impaired protein C (PC) system's function. The purpose of this study is to evaluate the association between severity of malaria and the EPCR genotypes as well as the soluble EPCR (SEPCR), and the study also addresses the physiological relevance of EPCR genetic polymorphism. In this study, we conducted meta-analysis on the eligible studies by comparing the frequency of EPCR rs867186-GG versus rs867186- GA and -AA genotype in SM, mild malaria (MM) or uncomplicated malaria (UM) patients and healthy individuals from Thailand, Uganda, Benin, Tanzania, and Ghana. We also determined the relationship between rs867186 genotype and SEPCR levels. Our results showed that the gene type of rs867186-GG is higher in MM/UM than in SM patients. SM patients carrying the rs867186-GG genotype have higher plasma soluble EPCR (sEPCR) levels than in rs867186-AG and rs867186-AA carriers. A significant difference is seen with the higher plasma sEPCR expression among MM/UM patients carrying the rs867186-AG genotype compared to those carrying rs867186-AA. Similarly, the rs867186-GG is associated with SEPCR level in healthy individuals. In conclusion, this meta-analysis demonstrates that pRBCs and EPCR interactions are associated with malaria severity, and treatments that block pRBC binding to EPCR via PfEMP1 CIDRa1 could be a potential therapy for SM.

\section{Introduction}

Severe malaria (SM) is defined as cerebral malaria (CM), severe malaria anemia (SMA), high levels of parasitemia, hypoglycemia, and increased creatinine, which is the leading cause of morbidity and mortality of patients with plasmodium falciparum (P. falciparum) infections $^{1-2}$. The sequestration of parasitized red blood cells (pRBC) to host microvasculature beds in a variety of organs and tissues, a characteristic pathological feature of SM, is caused by their binding to endothelial cells (known as cytoadherence) via $P$. falciparum erythrocyte membrane protein 1 (PfEMP1) ${ }^{3-9}$. PfEMP1 protein is encoded by approximate 60 var genes with each parasite expressing only one vargene at a time ${ }^{10}$. The extracellular portion of PfEMP1 is composed of cysteine-rich interdomain region (CIDR) and Duffy binding-like (DBL) domains ${ }^{11-13}$. N-terminal DBL-CIDR tandem is referred to as the semi-conserved protein head structure and is the most conserved extracellular region ${ }^{14}$. PfEMP1 with CIDRa1 domains is shown to bind endothelial protein $\mathrm{C}$ receptor (EPCR) ${ }^{11,15}$, while PfEMP1 with CIDRa2-6 domains binds to CD $36^{11,15}$, and PfEMP1 with DBL $\beta 5$ domains binds to intercellular adhesion molecule 1 (ICAM1) ${ }^{13,15}$. The parasite sequestration, which is associated with disease severity, is determined by the extent of PfEMP1 and the above host receptors binding ${ }^{16-18}$. As a result, pRBC binding to CD36 on platelets, monocytes, and some endothelial cells is considered as uncomplicated or mild malaria (UM/MM), whereas pRBC adhesion to ICAM-1 is considered as an indicator of SM ${ }^{16-18}$. Recently, Turner et al. discovered that EPCR interactions with PfEMP1 domain cassette 8 (DC8) and DC13 (both of which contain CIDRa1 domain) are correlated with $S M^{19}$. Following their study, several research groups further showed that the binding of CIDRa1 to EPCR competitively inhibits activated protein C (APC)'s binding to EPCR, inducing pro-apoptotic and pro-inflammatory process and concomitantly inhibiting cytoprotection, anticoagulation and barrier functions in the host ${ }^{20-22}$; ultimately, the binding of CIDRa1 to EPCR leads to SM including CM and SMA ${ }^{23}$. By using qRT-PCR to test different subsets of PfEMP1 vargenes on CM samples from Ugandan children, Shabani et al. demonstrated that the EPCR-binding PFEMP1 transcript levels are important for the development of SM ${ }^{12}$. They found that children with combined CM and SMA expressed the highest EPCR-binding PFEMP1 transcripts, noting that as disease severity decreases, the EPCRbinding PfEMP1 transcripts decrease as well ${ }^{12}$. Their findings about the association between the increased EPCR-binding PfEMP1 (var CIDRa1 domain) transcript and the severity of malaria indicate that EPCR-binding PfEMP1 transcript could be a novel therapeutic target for SM. The abundance of the transcripts increased with malaria severity; however, each individual subtype transcript did not vary remarkedly between patients, which suggests that all EPCR-binding CIDRa1 subtypes are required to be targeted to deplete $P$. falciparum sequestration in host blood circulation, in particular for sequestration in brain blood circulation ${ }^{11}$. Several lines of evidence support a correlation between the plasma EPCR levels and the genetic polymorphisms of EPCR gene, notably the EPCR rs867186-A/G genotype 1-

2,24-26. Soluble EPCR (SEPCR) are able to competitively bind to the available CIDRa1 domain and then restore cellular EPCR function by freeing the EPCR binding sites for APC. Due to a lack of systemic analysis of the relationships among EPCR genotypes, sEPCR levels, and 
malaria disease severity, in the present study, we will perform a meta-analysis based on related literatures to determine the clinical significance of EPCR gene in the pathogenesis of SM.

\section{Methods}

\section{Search strategy}

Pubmed, Medline, Web of Science, Scopus and Embase were searched until April 2019 using the following terms: "endothelial protein C receptor", "EPCR", "malaria", "severe malaria/complicated malaria/uncomplicated malaria", "cerebral malaria", "severe malaria anemia", "polymorphism" and 'clinical studies'. Articles screened were obtained by titles first, then by article abstracts. After exclusion of nonrelevant publications, the remaining papers were evaluated in the full text version depending on in- and exclusion criteria. All searched data were retrieved, and the language of publication was restricted to English.

\section{Selection criteria}

In this meta-analysis, we collected all eligible publications regarding the correlation between EPCR and the clinical outcomes and/or clinicopathological features in malaria patients. Studies indentified met the following the requirement of inclusion: (1) rs867186-A/G genotype was analyzed and evaluated using DNA from peripheral blood mononuclear cells; (2) articles that studied the correlation between rs867186-A/G genotype and malaria severity; (3) articles that studied SM using similar criteria, which was defined as the presence of one of the following signs: high P. falciparum parasitemia $(>100,000$ parasites/ $\mu \mathrm{l})$, hypoglycaemia (glucose level $<22 \mathrm{nmol} / \mathrm{l})$, severe anemia (hematocrit $<20 \%$ or hemoglobin $<7 \mathrm{~g} / \mathrm{dl}$ ), increased serum creatinine $(>3 \mathrm{mg} / \mathrm{dl}$ ), and unrousable coma (or a Blantyre Coma Score below 3 ) caused by malaria infection regardless of the other signs (cerebral malaria); and (4) studies with hazard ratio (HR) and 95 $\%$ confidence interval $(\mathrm{Cl})$ that can be estimated. The exclusion criteria include: (1) letters, case reports, reviews, editorials, conference abstracts, expert opinion; (2) studies without information on the genotype of the AA and AG alleles of rs867186 (rs867186-A/G genotype); and (3) all in vitro/ex vivo studies including cell culture and animal studies.

\section{Data extraction}

Two investigators independently extracted data from eligible studies. Disagreements were resolved through fully discussion until consensus was reached. The following data was identified and recorded for each study: year of publication, the first author's name, number of cases, sample source, rs867186-A/G genotype, and clinicopathological parameters. Data for study characteristics were collected and summarized, and the heterogeneity of each study was evaluated as well.

\section{Statistical analysis}

The data analysis was performed using the software Review Manager 5.3 (Cochrane Collaboration, Oxford, UK) and Stata 12.0 (Stata Corporation, TX, USA). Comparisons of dichotomous measures were conducted by pooled odds ratios (ORs). Heterogeneity was determined by a chi-square test with significance set at $\mathrm{P}<0.10$; the total variation among studies was determined by I square. The I square value is an estimate of variance due to between-study heterogeneity rather than chance (the Cochran $Q$ statistics). $P$ value of $<0.05$ was considered to be statistically significant. If there were heterogeneity among studies (when I square exceeds $50 \%$ ), we used a random effect model to pool the ORs; otherwise, a fixed effect model was selected.

The database search generated 28 articles. After initial screening, 25 full-text studies were retrieved for more detailed assessment. Eventually, 5 studies were identified for further meta-analysis (Fig. 1).

\section{Results}

\section{Identification of relevant studies}

Twenty-eight articles were identified and twenty-three of those were excluded due to non-original articles (review), laboratory studies, and studies irrelevant to the current analysis. Finally, there were 5 studies that met the selection criteria and were included in the final metaanalysis (Fig.1). 


\section{Study characteristics}

Five studies, published from 2014 to 2016 , were eligible for meta-analysis ${ }^{1-2,24-26}$. A total of 2995 SM, 487 uncomplicated malaria and 2105 healthy controls from Thailand, Uganda, Benin, Tanzania, and Ghana were enrolled. The characteristics are listed in Table 1. The definition criteria for SM of the 5 manuscripts is presented in Table 2

\section{3. rs867186 $A>G$ and the risk of malaria}

We compared the genotype frequencies of GG (rs867186-GG) versus AA (rs867186-AA) alleles of rs867186 in malaria patients and healthy individuals. We observed that the prevalence of rs867186-GG is not higher in healthy controls than in malaria patients. The pooled OR from 3 studies, including 2532 patients with malaria and 2105 healthy individuals, is shown in Fig. 2 (OR=0.92, $95 \%$ Cl=0.741.15, $P=0.49)$.

\section{Association of rs867186-G variant with higher plasma soluble EPCR (sEPCR) levels}

Two studies were eligible to analyze the relationship between PROCR gene, rs867186-G (GG or AG) variant, and plasma soluble EPCR levels. In the Beninese studies conducted by Moussiliou et al., no patient had the homozygous recessive genotype rs867186-GG. At convalescence, the overall analysis of genotype showed a higher plasma sEPCR levels among CM and SM patients carrying the rs867186-AG genotype compared with those carrying rs867186-AA as shown in Table 3 (297ng/ml vs. 158ng/ml, respectively; $\mathrm{P}=0.03)$. There is an upward trend of plasma EPCR levels among CM and SM patients carrying the rs867186-AG genotype compared with those carrying rs867186-AA $(P=0.17)$ at admission, but they are not significantly different ${ }^{24}$. The studies from Shabani ${ }^{26}$ showed that $C M$ and SMA patients carrying rs867186-GG or rs867186-AG genotype have higher sEPCR levels than rs867186-AA carriers (194ng/ml and $131 \mathrm{ng} / \mathrm{ml}$ vs. $84.5 \mathrm{ng} / \mathrm{ml}, \mathrm{P}=0.007$ and $\mathrm{P}<0.001$, respectively, Table 3 ). In addition, a clear difference was observed with higher plasma sEPCR levels among uncomplicated malaria patients carrying the rs867186-AG genotype compared to those carrying rs867186-AA $(161 \mathrm{ng} / \mathrm{ml}$ vs. $86.5 \mathrm{ng} / \mathrm{ml}, \mathrm{P}<0.001$, Table 3$)$. Similarly, the rs867186-GG was associated with higher sEPCR levels in healthy individuals (350ng/ml vs. $241 \mathrm{ng} / \mathrm{ml}, \mathrm{P}<0.006$, Table 3). Our results show the association between rs867186-G variant and higher circulating sEPCR levels both in SM patients including CM and SMA, and high $P$. falciparum parasitemia burden $(>250,000$ parasites/ $\mu$ l) 24,26 , and in $\mathrm{UM} / \mathrm{MM}$ and healthy individuals.

\section{Association of rs867186-GG genotype with protection of SM}

We analyzed the genotype frequencies of the GG alleles (rs867186-GG) versus AA and AG alleles (rs867186-AA+AG) of rs867186 in SM and uncomplicated/mild malaria (UM/MM) patients. We observed that the prevalence of rs867186-GG is higher in mild malaria than in SM patients. The pooled OR from 2 studies including 892 patients with SM and 437 mild malaria is shown in Fig. 3 (OR=0.36, $95 \%$ $\mathrm{Cl}=0.14-0.91, \mathrm{P}=0.03)$. The possible reason that the rs867186 genotypes distinguish between mild malaria and $\mathrm{SM}$ but not healthy controls and malaria patients (Fig.3) is due to healthy controls lacking the pressure exerted by parasites of the genus Plasmodium, which causes malaria infection.

\section{Sensitivity analyses and publication bias}

A sensitivity analysis was performed to assess the result stability. The results showed that the pooled ORs are not significantly changed, indicating the stability of the analytic results. The funnel plots are largely symmetric, indicating there are no publication biases in the meta-analysis of the role of rs867186-GG genotype in the SM (Fig. 4).

\section{Discussion}

The surface protein of EPCR was originally cloned in 1994. Multiple ligands of EPCR have been found, including PfEMP1, protein C (PC)/ activated protein C (APC), factor VIla, and a specific variant of the T-cell receptor. Under physiological conditions, Protein C is activated by the thrombin-thrombomodulin complex, and activated protein C (APC) then cleaves the protease-activated receptor (PAR1) at Arg46, which triggers anti-apoptotic, anti-inflammatory reaction to inhibit thrombin production and stabilize endothelial barrier function $21,27-28$. In SM, PfEMP1 binding to EPCR, which blocks APC to EPCR, compromises this negative feedback, inducing apoptosis, inflammation, loss 
of endothelial barrier function, localized vascular leak, and coagulation. In addition, PfEMP1 binding to EPCR, which causes microvascular congestion and obstruction, is another reason to exacerbate malaria $11,13,22$.

Each Plasmodium falciparum genome holds about 60 PfEMP1 encoding var genes. DC13-containing PfEMP1 variants have the ability to bind to both the EPCR and ICAM-1 ${ }^{22}$. Both ICAM-1 ${ }^{29-31}$ and EPCR ${ }^{9,32-35}$ are implicated in CM, ICAM-1: PfEMP1 binding and resetting have been identified as one of the virulence factors ${ }^{36}$, while EPCR-binding parasites dominate host infections with limited malaria immunity 9,32-35. sEPCR is shed from endothelial cells, and the addition of soluble recombinant EPCR inhibits the binding between DC13- and DC8containing PfEMP1 variants and endothelial cells ${ }^{19}$, which in turn is associated with a higher risk of venous thrombosis ${ }^{19,37}$. Thus, it seems that human polymorphism in EPCR may be a balanced polymorphism protecting individuals against SM at the expense of a higher risk for thrombotic disease ${ }^{19,37}$.

To assess the effect of the polymorphism on the risk of SM, we analyzed genotyped patients with mild malaria and SM on rs867186-GG of previous publications. We found the rs867186-GG genotype appear significantly more frequently in patients with mild malaria than those with SM ( $P=0.03$ in Fig.3). However, when we compared the genotypes of rs867186-GG versus rs867186-AA in malaria patients and healthy individuals, the frequency of the rs867186-GG in malaria patients and healthy controls is very similar ( $p=0.90$ in Fig.2). This was not caused by studies bias, as the heterogeneity is calculated as $\mathrm{I}^{2}=0$ (Fig.2), which implies that we can take a fixed effect model to pool the ORs other than a random effect model. Malaria is a mosquito-borne disease that can be prevented. The genotype difference of the GG and AA alleles of rs867186 is likely due to pressure exerted by parasites of the genus Plasmodium that cause malaria. Thus, comparing healthy controls that lack of these pressure with any disease status is unnecessary. This is a possible reason that these genotypes distinguish between mild and SM but not between healthy controls and malaria patients. The studies from Hansson et al. ${ }^{1}$ and Schuldt et al. ${ }^{25}$ reported that the genotype rs867186-GG does not have a protective role in malaria patients compared to healthy controls. However, they did not compare rs867186-GG genotype difference between SM and MM.

A soluble EPCR (sEPCR) is produced by metalloprotease activity and circulates in plasma ${ }^{19}$. The relationship between the polymorphisms in EPCR and plasma SEPCR levels has been conducted in patients with thrombosis to evaluate the risk of venous thrombosis by Mdina et al. ${ }^{38}$. Their data indicate that individuals carrying some specific genotypes have high sEPCR and APC levels, thus having lower risk of venous thromboembolism ${ }^{38}$. However, no systemic analysis has been made in malaria patients regarding the EPCR genotype and plasma EPCR levels. When we examined the relationship between the rs867186 polymorphisms and plasma sEPCR evaluating risk of SM, we found that the carriers of the rs867186-GG genotype have significantly higher sEPCR levels than those with the AG and AA genotype in SM, carriers of the rs867186-AG genotype have significantly higher sEPCR levels than those with AA genotype in uncomplicated malaria, and carriers of the rs867186-GG genotype have significantly higher sEPCR levels than those with AG genotype in healthy individuals (Table 3). These results support that the rs867186 GG genotype is associated with elevated sEPCR levels in SM (Table

3). The loss of endothelial protein C receptor link coagulation and inflammation to parasite sequestration in $\mathrm{CM} 26,39$, and rs867186-GG is associated with increased soluble EPCR and can potentially mediate protection against SM.

Two studies involving adult malaria patients ${ }^{2,12}$ revealed that the EPCR rs867186-G allele could mediate protection against SM, while the other three studies involving child malaria patients showed GPCR gene variants are not associated with SM ${ }^{1,25}$ or increased mortality among children with $\mathrm{CM}^{24}$. Although they utilized a similar criteria for SM, adult and child malaria may have different pathophysiology. Once more studies in this field are available in future, the EPCR polymorphism study should be conducted on adult and child malaria separately.

In conclusion, this meta-analysis summarizes previous observations and indicates the EPCR rs867186-GG genotypes is associated with increased soluble EPCR and may have an important role in SM. pRBCs bind to EPCR on endothelial cells and block APC's access to EPCR, inducing pro-apoptotic, pro-inflammatory, and loss of local vascular barrier. Targeting EPCR-binding PfEMP1 can potentially reverse $P$. falciparum sequestration in host blood circulation, and in particular for sequestration in brain blood circulation, which could be an effective intervention to prevent SM. In the future, more studies are needed to assess the adhesive abilities of PfEMP1 expressed in isolation from SM, CM patients, and even pregnant women with malaria. In addition, the mechanism of human polymorphism in EPCR protecting individuals against SM and balancing the increasing risk of thrombotic disease is also worthy of exploration.

\section{Declarations}

\section{Competing interest}

Page 5/11 


\section{Authors' contribution}

LY and ML contributed substantially to the study design, acquisition of data, analysis, and interpretation of data. RX and SG contributed substantially to the acquisition, analysis, interpretation of data, and performed the statistical analysis. $L Y$ and ML have been involved in the drafting process and critical revision of the article for important intellectual content. The corresponding authors had full access to all data and the final responsibility for the decision to submit the article for publication. All authors read and approved the final manuscript.

\section{Acknowledgement}

We thank studies' participants.

\section{Supplementary material}

PRISMA Check list

\section{References}

1. Hansson HH, Turner L, Moller L, et al. Haplotypes of the endothelial protein $C$ receptor (EPCR) gene are not associated with severe malaria in Tanzania. Malaria journal. 2015;14:474.

2. Naka I, Patarapotikul J, Hananantachai H, Imai H, Ohashi J. Association of the endothelial protein C receptor (PROCR) rs867186-G allele with protection from severe malaria. Malaria journal. 2014;13:105.

3. Moxon CA, Grau GE, Craig AG. Malaria: modification of the red blood cell and consequences in the human host. British journal of haematology. 2011;154(6):670-679.

4. Nielsen MA, Staalsoe T, Kurtzhals JA, et al. Plasmodium falciparum variant surface antigen expression varies between isolates causing severe and nonsevere malaria and is modified by acquired immunity. Journal of immunology (Baltimore, Md : 1950). 2002;168(7):34443450 .

5. Jensen AT, Magistrado P, Sharp S, et al. Plasmodium falciparum associated with severe childhood malaria preferentially expresses PfEMP1 encoded by group A var genes. The Journal of experimental medicine. 2004;199(9):1179-1190.

6. Bull PC, Pain A, Ndungu FM, et al. Plasmodium falciparum antigenic variation: relationships between in vivo selection, acquired antibody response, and disease severity. The Journal of infectious diseases. 2005;192(6):1119-1126.

7. Rottmann M, Lavstsen T, Mugasa JP, et al. Differential expression of var gene groups is associated with morbidity caused by Plasmodium falciparum infection in Tanzanian children. Infection and immunity. 2006;74(7):3904-3911.

8. Kyriacou HM, Stone GN, Challis RJ, et al. Differential var gene transcription in Plasmodium falciparum isolates from patients with cerebral malaria compared to hyperparasitaemia. Molecular and biochemical parasitology. 2006;150(2):211-218.

9. Lavstsen T, Turner L, Saguti F, et al. Plasmodium falciparum erythrocyte membrane protein 1 domain cassettes 8 and 13 are associated with severe malaria in children. Proceedings of the National Academy of Sciences of the United States of America. 2012;109(26):E1791-1800.

10. Scherf A, Lopez-Rubio JJ, Riviere L. Antigenic variation in Plasmodium falciparum. Annual review of microbiology. 2008;62:445-470.

11. Mkumbaye SI, Wang CW, Lyimo E, et al. The Severity of Plasmodium falciparum Infection Is Associated with Transcript Levels of var Genes Encoding Endothelial Protein C Receptor-Binding P. falciparum Erythrocyte Membrane Protein 1. Infection and immunity. 2017;85(4). 
12. Shabani E, Hanisch B, Opoka RO, Lavstsen T, John CC. Plasmodium falciparum EPCR-binding PfEMP1 expression increases with malaria disease severity and is elevated in retinopathy negative cerebral malaria. BMC medicine. 2017;15(1):183.

13. Tuikue Ndam N, Moussiliou A, Lavstsen T, et al. Parasites Causing Cerebral Falciparum Malaria Bind Multiple Endothelial Receptors and Express EPCR and ICAM-1-Binding PfEMP1. The Journal of infectious diseases. 2017;215(12):1918-1925.

14. Su XZ, Heatwole VM, Wertheimer SP, et al. The large diverse gene family var encodes proteins involved in cytoadherence and antigenic variation of Plasmodium falciparum-infected erythrocytes. Cell. 1995;82(1):89-100.

15. Mustaffa KMF, Storm J, Whittaker M, Szestak T, Craig AG. In vitro inhibition and reversal of Plasmodium falciparum cytoadherence to endothelium by monoclonal antibodies to ICAM-1 and CD36. Malaria journal. 2017;16(1):279.

16. Newbold C, Warn P, Black G, et al. Receptor-specific adhesion and clinical disease in Plasmodium falciparum. The American journal of tropical medicine and hygiene. 1997;57(4):389-398.

17. Ochola LB, Siddondo BR, Ocholla $\mathrm{H}$, et al. Specific receptor usage in Plasmodium falciparum cytoadherence is associated with disease outcome. PloS one. 2011;6(3):e14741.

18. Wassmer SC, Lepolard C, Traore B, Pouvelle B, Gysin J, Grau GE. Platelets reorient Plasmodium falciparum-infected erythrocyte cytoadhesion to activated endothelial cells. The Journal of infectious diseases. 2004;189(2):180-189.

19. Turner L, Lavstsen T, Berger SS, et al. Severe malaria is associated with parasite binding to endothelial protein $\mathrm{C}$ receptor. Nature. 2013;498(7455):502-505.

20. Pendurthi UR, Rao LVM. Endothelial cell protein C receptor-dependent signaling. Current opinion in hematology. 2018;25(3):219-226.

21. Bernabeu M, Smith JD. EPCR and Malaria Severity: The Center of a Perfect Storm. Trends in parasitology. 2017;33(4):295-308.

22. Lennartz F, Adams Y, Bengtsson A, et al. Structure-Guided Identification of a Family of Dual Receptor-Binding PfEMP1 that Is Associated with Cerebral Malaria. Cell host \& microbe. 2017;21(3):403-414.

23. Mosnier LO, Lavstsen T. The role of EPCR in the pathogenesis of severe malaria. Thrombosis research. $2016 ; 141$ Suppl 2:S46-49.

24. Moussiliou A, Alao MJ, Denoeud-Ndam L, et al. High plasma levels of soluble endothelial protein C receptor are associated with increased mortality among children with cerebral malaria in Benin. The Journal of infectious diseases. 2015;211(9):1484-1488.

25. Schuldt K, Ehmen C, Evans J, et al. Endothelial protein C receptor gene variants not associated with severe malaria in ghanaian children. PloS one. 2014;9(12):e115770.

26. Shabani E, Opoka RO, Bangirana P, et al. The endothelial protein C receptor rs867186-GG genotype is associated with increased soluble EPCR and could mediate protection against severe malaria. Scientific reports. 2016;6:27084.

27. Mohan Rao LV, Esmon CT, Pendurthi UR. Endothelial cell protein C receptor: a multiliganded and multifunctional receptor. Blood. 2014;124(10):1553-1562.

28. Deane R, LaRue B, Sagare AP, Castellino FJ, Zhong Z, Zlokovic BV. Endothelial protein C receptor-assisted transport of activated protein C across the mouse blood-brain barrier. J Cereb Blood Flow Metab. 2009;29(1):25-33.

29. Howell DP, Levin EA, Springer AL, et al. Mapping a common interaction site used by Plasmodium falciparum Duffy binding-like domains to bind diverse host receptors. Mol Microbiol. 2008;67(1):78-87.

30. Bengtsson A, Joergensen L, Rask TS, et al. A novel domain cassette identifies Plasmodium falciparum PfEMP1 proteins binding ICAM-1 and is a target of cross-reactive, adhesion-inhibitory antibodies. J Immunol. 2013;190(1):240-249.

31. Joergensen L, Bengtsson DC, Bengtsson A, et al. Surface co-expression of two different PfEMP1 antigens on single plasmodium falciparum-infected erythrocytes facilitates binding to ICAM1 and PECAM1. PLoS Pathog. 2010;6(9):e1001083.

32. Bernabeu M, Danziger SA, Avril M, et al. Severe adult malaria is associated with specific PfEMP1 adhesion types and high parasite biomass. Proc Natl Acad Sci U S A. 2016;113(23):E3270-3279.

Page $7 / 11$ 
33. Jespersen JS, Wang CW, Mkumbaye SI, et al. Plasmodium falciparum var genes expressed in children with severe malaria encode CIDRalpha1 domains. EMBO Mol Med. 2016;8(8):839-850.

34. Cham GK, Turner L, Lusingu J, et al. Sequential, ordered acquisition of antibodies to Plasmodium falciparum erythrocyte membrane protein 1 domains. J Immunol. 2009;183(5):3356-3363.

35. Turner L, Lavstsen T, Mmbando BP, et al. IgG antibodies to endothelial protein C receptor-binding cysteine-rich interdomain region domains of Plasmodium falciparum erythrocyte membrane protein 1 are acquired early in life in individuals exposed to malaria. Infect Immun. 2015;83(8):3096-3103.

36. Rowe JA, Claessens A, Corrigan RA, Arman M. Adhesion of Plasmodium falciparum-infected erythrocytes to human cells: molecular mechanisms and therapeutic implications. Expert Rev Mol Med. 2009;11:e16.

37. Angchaisuksiri P. Coagulopathy in malaria. Thrombosis research. 2014;133(1):5-9.

38. Medina P, Navarro S, Estelles A, et al. Contribution of polymorphisms in the endothelial protein C receptor gene to soluble endothelial protein C receptor and circulating activated protein C levels, and thrombotic risk. Thromb Haemost. 2004;91(5):905-911.

39. Moxon CA, Wassmer SC, Milner DA, Jr., et al. Loss of endothelial protein C receptors links coagulation and inflammation to parasite sequestration in cerebral malaria in African children. Blood. 2013;122(5):842-851.

\section{Tables}

Table 1. Basic characteristics of the included studies

\begin{tabular}{|c|c|c|c|c|c|c|c|c|c|c|c|c|c|}
\hline \multirow[t]{3}{*}{ Study } & \multirow[t]{3}{*}{ Year } & \multirow[t]{3}{*}{ Population } & \multirow{2}{*}{\multicolumn{3}{|c|}{$\begin{array}{l}\text { SM } \\
\text { No. } \\
\text { of } \\
\text { cases }\end{array}$}} & \multirow{2}{*}{\multicolumn{3}{|c|}{$\begin{array}{l}\text { MM } \\
\text { No. } \\
\text { of } \\
\text { cases }\end{array}$}} & \multirow{2}{*}{\multicolumn{2}{|c|}{$\begin{array}{l}\text { HC } \\
\text { No. } \\
\text { of } \\
\text { cases }\end{array}$}} & & \multirow[t]{3}{*}{ SNP } & \multirow{3}{*}{$\begin{array}{l}\text { Conclusion } \\
\text { Reported }\end{array}$} \\
\hline & & & & & & & & & & & & & \\
\hline & & & GG n & $\begin{array}{l}\mathrm{GA} \\
\mathrm{n}\end{array}$ & $A A n$ & GG n & $\begin{array}{l}\mathrm{GA} \\
\mathrm{n}\end{array}$ & $\begin{array}{l}\mathrm{AA} \\
\mathrm{n}\end{array}$ & GG n & $\begin{array}{l}\mathrm{GA} \\
\mathrm{n}\end{array}$ & $\begin{array}{l}\mathrm{AA} \\
\mathrm{n}\end{array}$ & & \\
\hline Naka & 2014 & Thailand & 5 & 94 & 242 & 16 & 88 & 262 & & & & rs867186 & Protective \\
\hline Schuldt & 2014 & Ghana & 108 & & 1797 & & & & 110 & & 1756 & rs867186 & $\begin{array}{l}\text { No } \\
\text { association }\end{array}$ \\
\hline Hansson & 2015 & Tanzania & 8 & 68 & & & & & 7 & 60 & & rs867186 & $\begin{array}{l}\text { No } \\
\text { association }\end{array}$ \\
\hline Moussiliou & 2015 & Benin & & 19 & 103 & & 9 & 41 & & & & rs867186 & $\begin{array}{l}\text { High } \\
\text { mortality }\end{array}$ \\
\hline Shabani & 2016 & Uganda & 3 & 102 & 446 & 0 & 14 & 57 & 7 & 31 & 134 & rs867186 & Protective \\
\hline
\end{tabular}

Table 2. Definition criteria for severe malaria

\begin{tabular}{|c|c|c|c|c|c|}
\hline Study & Severe malaria & & & & \\
\hline Naka 2014 & High $P f$ & SMA & hypoglycaemia & High serum creatinine & $\mathrm{CM}$ \\
\hline Schuldt 2014 & High $P f$ & SMA & & & $\mathrm{CM}$ \\
\hline Hansson 2015 & High $P f$ & SMA & & & $\mathrm{CM}$ \\
\hline Moussiliou 2015 & High $P f$ & SMA & & & $\mathrm{CM}$ \\
\hline Shabani 2016 & & SMA & & & $\mathrm{CM}$ \\
\hline
\end{tabular}

High Pf: >100,000 parasites/ $\mu$ l

SMA: severe anemia: hematocrit $(\mathrm{HCT})<20 \%$, hemoglobin $(\mathrm{Hb})<7 \mathrm{~g} / \mathrm{dl}$ 
CM: cerebral malaria

High serum creatinine: serum creatinine $>3 \mathrm{mg} / \mathrm{dl}$

Hypoglycaemia: glucose $<22 \mathrm{nmol} / \mathrm{I}$

Table 3: rs867186-G variant is associated with higher sEPCR levels in severe malaria

\begin{tabular}{|c|c|c|c|c|c|}
\hline \multirow[t]{2}{*}{ Study } & \multirow[t]{2}{*}{ Stages } & \multirow{2}{*}{$\begin{array}{l}\text { Genotype } \\
\text { (n=number) }\end{array}$} & Plasma levels of sEPCR & \multirow[b]{2}{*}{ Interquartile range (IQR) } & \multirow[b]{2}{*}{$P$ value } \\
\hline & & & $\begin{array}{l}\text { Median } \\
(\mathrm{ng} / \mathrm{ml})\end{array}$ & & \\
\hline Moussiliou & \#CM+\#\#\#SM & AA & 158 & $131-211$ & \\
\hline 2015 & Convalescence & $A G$ & 297 & $174-383$ & $P=0.03$ \\
\hline Shabani & $\mathrm{CM}+\# \# S M A$ & $\mathrm{AA}(\mathrm{n}=390)$ & 84.5 & $65.7-104$ & \\
\hline \multicolumn{6}{|l|}{2016} \\
\hline & & $A G(n=91)$ & 131 & $107-170$ & ${ }^{*} P<0.001$ \\
\hline & & $G G(n=3)$ & 194 & $104-211$ & $* * P=0.007$ \\
\hline & & & & & $\star \star * P=0.71$ \\
\hline & \multirow[t]{2}{*}{ §UM } & $\mathrm{AA}(\mathrm{n}=25)$ & 86.5 & $75.4-113$ & \\
\hline & & $A G(n=13)$ & 161 & $142-164$ & $P<0.001$ \\
\hline & \multirow[t]{2}{*}{ §§Healthy CC } & $\mathrm{AA}(\mathrm{n}=79)$ & 98.4 & $87.8-121$ & \\
\hline & & $A G(n=25)$ & 241 & $203-288$ & \\
\hline & & GG $(n=6)$ & 350 & $319-380$ & $P<0.006$ \\
\hline
\end{tabular}

${ }^{\star} \mathrm{AG}$ compared with $\mathrm{AA}$, ** $\mathrm{GG}$ compared with $\mathrm{AA}$, *** $\mathrm{GG}$ compared with $A G$

\#CM (cerebral malaria), \#\#SMA (severe malaria anemia), \#\#\#SM: severe malaria: >250,000 parasites/ $\mu \mathrm{l}$, or SMA

§UM: uncomplicated malaria, §§̧ Healthy CC: healthy community controls

\section{Figures}




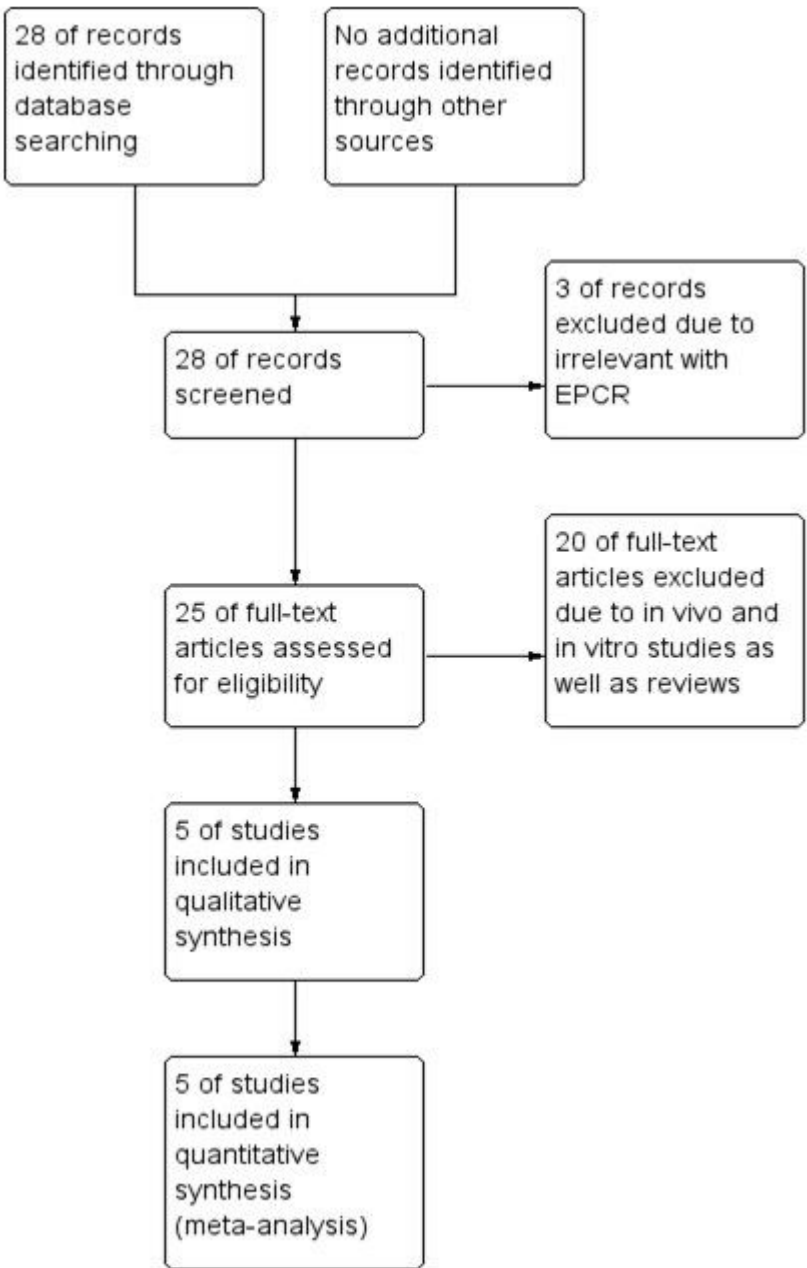

\section{Figure 1}

Flow chart of the study. Among twenty-eight publications identified by the search method, twenty-three of those were excluded due to exclusion criteria of laboratory studies, non-original articles, lack of matched controls or studies irrelevant to the current analysis. Five studies were included in this meta-analysis.

\section{Malaria Healthy Controls Odds Ratio Odds Ratio}

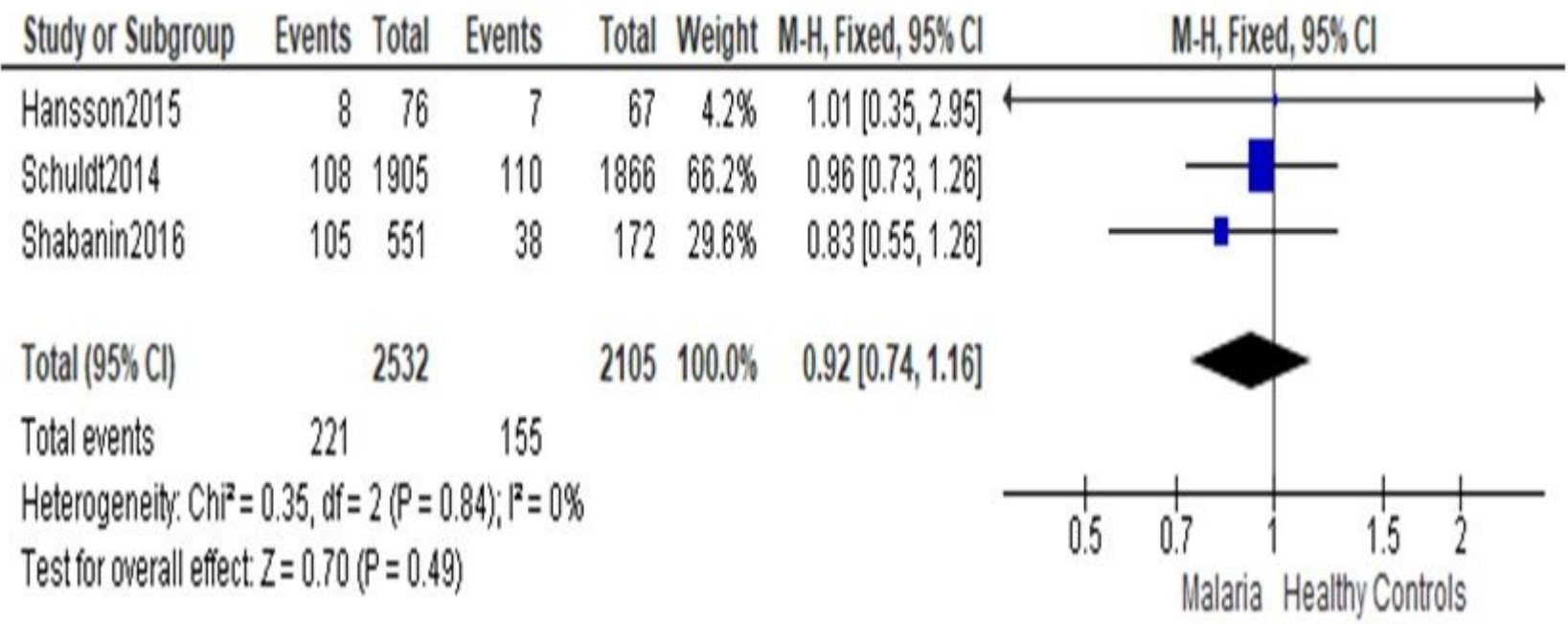

\section{Figure 2}


Forest plot for the risk of malaria and the EPCR polymorphism in the genotype of rs867186 GG vs. AA. The frequency of rs867186-GG versus rs867186-AA in malaria patients and healthy individuals was compared. The prevalence of rs867186-GG is not higher in healthy controls than in SM patients. The pooled OR from 3 studies, including 2532 patients with malaria and 2105 healthy individuals, is shown (OR=0.92 95\% Cl=0.74-1.16, $\mathrm{P}=0.49)$.

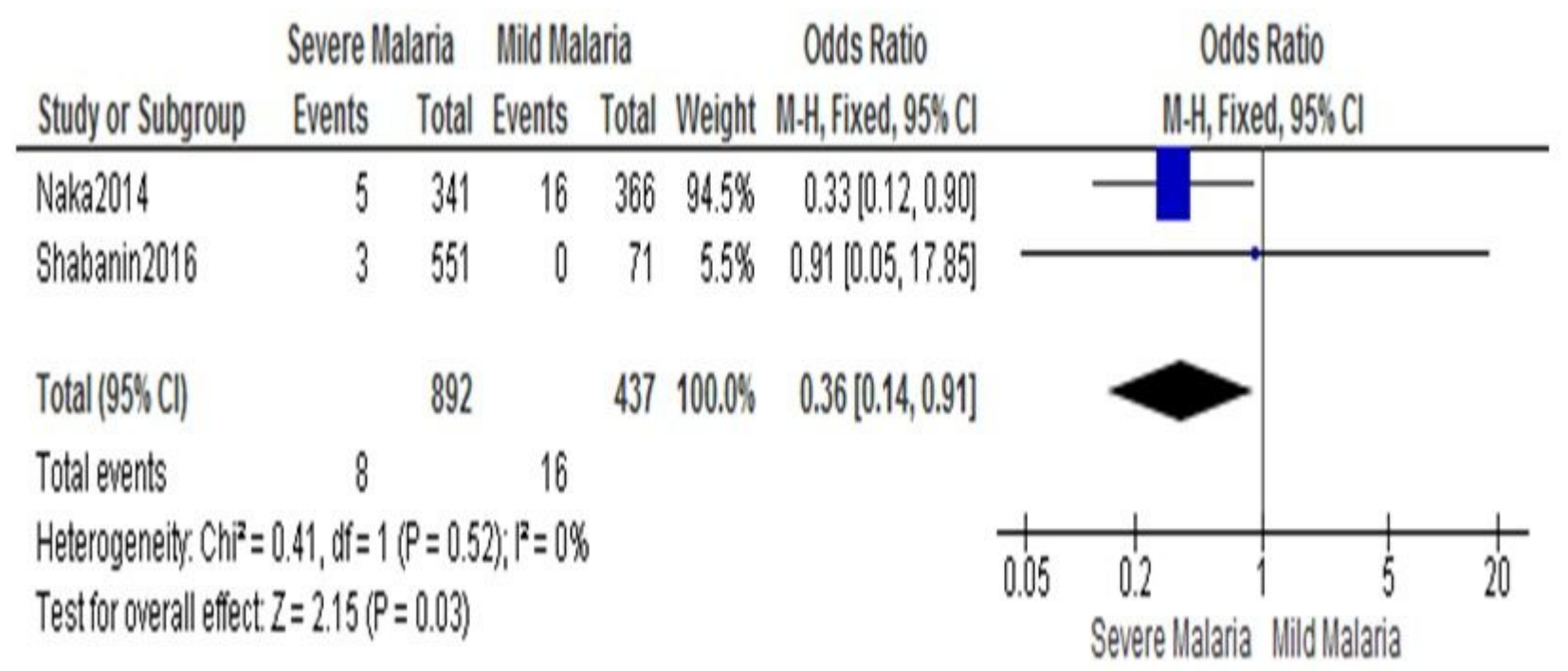

\section{Figure 3}

Forest plot for the protection of SM and the EPCR polymorphism in the genotype of rs $867186 \mathrm{GG}$ vs. AG and AA. The frequency of rs867186-GG versus rs867186-AA and AG was analyzed in SM patients and uncomplicated/mild malaria. The frequency of rs867186-GG is higher in mild malaria than in SM patients. The pooled OR from 2 studies, including 892 patients with SM and 437 mild malaria, is shown (OR=0.36, 95\% $\mathrm{Cl}=0.14-0.91, \mathrm{P}=0.03)$.

A.

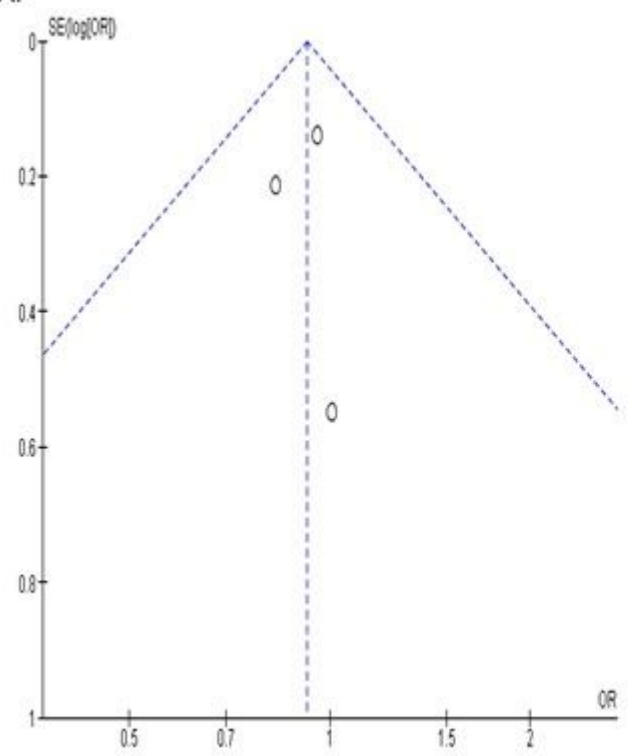

B.

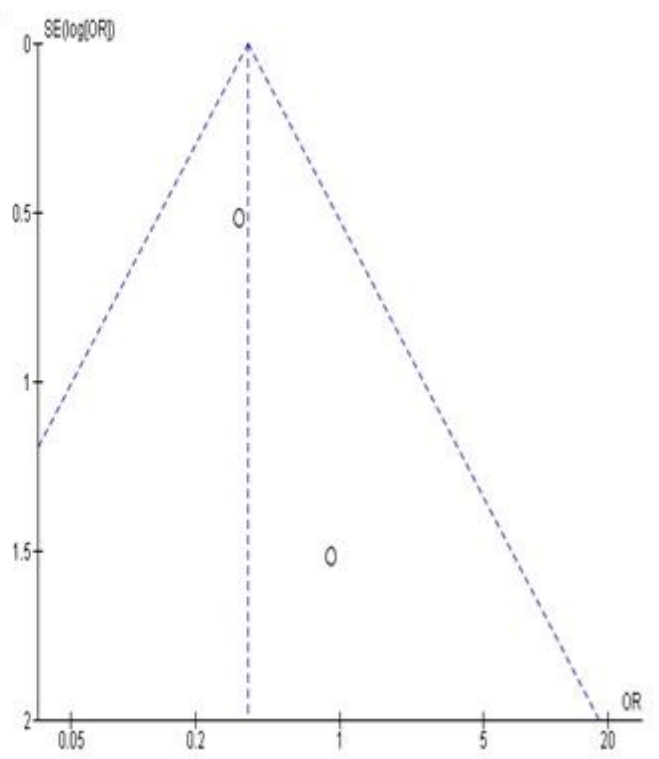

Figure 4

Funnel plot of publication bias in the meta-analysis of rs867186 genotype. The funnel plots are largely symmetric, suggesting there are no publication biases in the meta-analysis of the role of rs867186-GG genotype in the SM. 